\title{
Computational Hemodynamics Analysis of Intracranial Aneurysms Treated with Flow Diverters: Correlation with Clinical Outcomes
}

\author{
W. Chong, Y. Zhang, Y. Qian, L. Lai, G. Parker, and K. Mitchell
}

on

\begin{abstract}
BACKGROUND AND PURPOSE: Recent studies have shown promising results regarding intracranial aneurysms treated with flow diverters. However, these have had adverse effects, including delayed aneurysm occlusion, posttreatment symptoms, and rupture. The hemodynamic profiles of aneurysms treated with flow diverters were analyzed to determine the ones associated with successful and failed treatments.
\end{abstract}

MATERIALS AND METHODS: Patient-specific computational fluid dynamics were used to simulate hemodynamic profiles, including the presence of jet flow, energy loss, volume flow, and wall shear stress in 4 successful occlusions of aneurysms and 4 failed cases after flow-diverter deployment. In these 4 failed cases, hemodynamic profiles were examined again after a hypothetic second intervention. This involved replacing the failed flow diverter with a hypothetic optimally deployed flow diverter or simulated placement of a second flow diverter within the first (double hypothetic optimally deployed).

RESULTS: Where successful occlusions were achieved, a marked obliteration of jet flow was observed. Flow entering the aneurysm sac was diverted via the center of the flow diverter and joined smoothly with the continuation of flow leaving the aneurysm sac into the parent arteries. These observations were supplemented by a reduction in the other hemodynamic profiles. Aneurysm neck geometry might influence the efficacy of the flow diverter.

CONCLUSIONS: Hemodynamic indices, as calculated by using computational fluid dynamics techniques, have close correlation with flow-diverter treatment outcome. Computational fluid dynamics could be potentially useful as a planning tool for neurointerventionists by simulating an optimized flow-diverter deployment strategy before the procedure and evaluating posttreatment outcome.

ABBREVIATIONS: CFD = computational fluid dynamics; $\mathrm{EL}=$ energy loss; $\mathrm{FD}=$ flow diverter; $\mathrm{HOFD}=$ hypothetic optimally deployed FD; $\mathrm{VF}=\mathrm{volume}$ flow; WSS $=$ wall shear stress

D espite the availability of biologically coated aneurysm coils, the Onyx Liquid Embolic System (ev3, Irvine, California), and balloon- and stent-assisted coiling techniques, wide-neck large and giant aneurysms are very difficult and sometimes impossible to treat by endovascular means, due to significant residual and recurrence rates and unsatisfactory patient outcomes. ${ }^{1-3}$

Received December 28, 2012; accepted after revision April 5, 2013.

From the Monash Medical Centre (W.C.), Melbourne, Victoria, Australia; Australian School of Advanced Medicine (Y.Z., Y.Q., L.L.), Macquarie University, New South Wales, Australia; Royal Prince Alfred Hospital (G.P.), Sydney, New South Wales, Australia; and Royal Brisbane Hospital (K.M.), Brisbane, Queensland, Australia. This work was partially supported by the Australian Research Council Discovery Grant DP110102985.

Please address correspondence to Yi Qian, MD, Australian School of Advanced Medicine, Macquarie University, 2 Technology Place, North Ryde 2109, NSW, Australia; e-mail: yi.qian@mq.edu.au

- Indicates open access to non-subscribers at www.ajnr.org

三 Indicates article with supplemental on-line table

http://dx.doi.org/10.3174/ajnr.A3790
Surgical management is likewise unsatisfactory. The International Study of Unruptured Intracranial Aneurysms reported a poor 1 -year patient outcome rate of up to $34 \%$ for large and giant aneurysms. ${ }^{4}$ Flow diverters (FDs) have provided a promising new method for the endovascular reconstruction of these types of aneurysms. ${ }^{5,6}$ These are fine mesh stents that divert flow away from the aneurysm sac, thereby promoting progressive thrombosis and occlusion of the aneurysm without further addition of coils or other embolic material. Currently there are 2 devices on the market, the Pipeline Embolization Device (Covidien, Irvine, California) and Silk (Balt, Montmorency, France). Long-term results are not yet available, but current experience is promising. The timeframe for complete occlusion of an aneurysm is unknown, but recent series have documented 6-month occlusion rates of $89.0 \%-93.3 \% .^{7}$ Of more concern are reports of delayed parenchymal or subarachnoid hemorrhage following FD treatment. These have been estimated to be up to $1.75 \%$ for the Pipeline Embolization Device and $0.8 \%$ for Silk. ${ }^{7}$ Hemodynamic factors and aggres- 
sive thrombus-associated autolysis of the aneurysm wall have been implicated. ${ }^{8,9}$

Computational fluid dynamics (CFD) analysis has been applied in the study of the hemodynamics of aneurysm growth and rupture, as well as various types of stents, including FDs. ${ }^{10-15}$ The objective of this study was to analyze the hemodynamic profiles involved in successful and failed treatment by comparing 4 aneurysm cases with successful treatment outcome against 4 failed cases, through the use of CFD simulation.

\section{MATERIALS AND METHODS}

\section{Patients and Imaging Data}

After institutional ethics approval was given, 4 successful cases (patients 1-4) and 4 failed cases (patients 5-8) were studied (Online Table). A successful case was defined as a complete angiographic exclusion of the aneurysm from the circulation within 6 months. Failed cases were defined as exhibiting persistent residual aneurysms after 6 months, posttreatment rupture, and the development of increased symptoms and associated perianeurysmal MR imaging brain changes. The average aneurysm size was 4.2 $\mathrm{mm}$. All 8 patients were initially treated with a Silk FD. Each Silk $\mathrm{FD}$, regardless of size, is composed of forty $30-\mu$ nitinol and eight $50-\mu \mathrm{m}$ platinum microfilaments; with pore sizes of $110-250$ $\mu .^{7,11}$ For the successful cases, patients $1-4$, the aneurysms were completely occluded by 6 months. For the failed cases 5 and 6 , persistent residual aneurysms were observed at 6 months. Patient 7 developed increasing headaches and "blistering" of the aneurysm wall and brain edema on MR imaging at 3 months. Patients 6 and 7 were retreated with insertion of another FD (Silk) within the first one (double FD) at 6 months and 3 months, respectively. For patient 8 , the aneurysm ruptured at 3 and a half months following FD implantation. All patients had aspirin and clopidogrel for 3 months and continued on aspirin afterward.

\section{Vascular and Flow-Diverter Models}

Commercially available packages, Mimics (Materialise, Leuven, Belgium) and CFX 13.0 (ANSYS, Canonsburg, Pennsylvania), were used to perform patient-specific CFD simulation by using fine DICOM axial sections derived from DSA or CTA. In successful cases, the optimally deployed FD was simulated as a porous media layer, $0.1 \mathrm{~mm}$ thick, with a flow resistance equivalent to the actual FD, as previously described. ${ }^{11}$ Validation tests were performed to determine the flow-resistance parameters, and our preliminary study has been published. ${ }^{16}$ We calculated the following hemodynamic parameters: energy loss (EL), wall shear stress (WSS), and volume flow (VF) into the aneurysm sac.

EL is expressed as

1) $E L=E_{\text {in }}-E_{\text {out }}=\sum_{i=1}^{\text {nin }} \dot{m}_{i, \text { in }}\left(P_{i, \text { in }} / \rho+\frac{1}{2} u_{i, \text { in }}{ }^{2}\right)-$

$$
\sum_{i=1}^{\text {nout }} \dot{m}_{i, \text { out }}\left(P_{i, \text { out }} / \rho+\frac{1}{2} u_{i, \text { out }}^{2}\right)
$$

where $\dot{m}$ denotes mass flow rate; $P$, static pressure; and $u$, in- or output velocity as denoted by the subscripts $i=1,2,3, u_{1}, u_{2}, u_{3}$.
VF rate is calculated as the summation of blood volume flowing from the artery into the aneurysm sac via the aneurysm neck.

WSS is expressed as

2)

$$
W S S=\mu \dot{u},
$$

where $\mu$ is the dynamic viscosity of blood and $\dot{u}$ is the velocity gradient at the aneurysm wall.

The jet flow patterns were graphically represented and interpretations were made from these.

To demonstrate that EL and VF are not substantially affected by variations in inflow conditions, we tested the inflow condition at the ICA under 7 flow rates, 80, 100, 125, 150, 175, 200, and 250 $\mathrm{mL} / \mathrm{minute}$, for EL and VF for all patients. There were 8 patients, each tested with 7 flow rates, giving a total sample size of 56, resulting in 28 samples for each of the successful and unsuccessful cohorts. $t$ tests were performed between these 2 groups.

\section{Hypothetic Optimally Deployed Flow Diverters and Double HOFD Simulation}

The porosity resistance was zeroed at the proximal portion of the FD to represent the uncovered "gap" at the aneurysm inflow of the malpositioned FD (patient 6), and it was reduced by half to simulate the stretched FD (patient 8 ). The porosity of the optimally deployed FDs in the failed cases 5 and 7 was not altered. The porosity characteristics, implanted positions of the FD, and FD conditions that pertain to parent arteries were optimally modified under a "what-if" condition for the failed cases. In this study, hypothetic optimally deployed (HOFD) flow diverters were simulated to substitute for the malpositioned and stretched FD for both patients 6 and 8 . For all unsuccessful procedures, hypothetic placement of a second FD inside the first one (double HOFD) was simulated by increasing the porosity resistance by 10 -fold. ${ }^{16}$ The HOFD and double HOFD simulations were performed under the average inflow condition at $150 \mathrm{~mL} / \mathrm{min}$.

In this study, the resulting values of EL and VF at the initial FD, HOFD, and double HOFD were all expressed as a percentage of the values obtained under conditions without FDs.

\section{RESULTS}

\section{Jet Flow Observation}

A common flow pattern for all 8 cases before deployment of the FD is a broad jet flow passing through and into the aneurysm sac. Figure 1 depicts the flow patterns of pre- and post-FD treatment. In the successful case (patient 1), before FD deployment, the jet flow impacted the aneurysm surface (Fig $1 A$ ). Following FD deployment, however, the jet flow speed significantly decreased in the aneurysm sac. In addition, the flow direction was diverted to the center of the FD with marked stasis at the periphery of the aneurysm sac (Fig 1B). This favorable flow pattern (reduction of jet flow speed, central diversion, and peripheral stasis) was observed in all successful cases. However, in the failed case (patient 6 ), the original jet flow pattern persisted despite the deployment of the FD stent. The jet flow speed after FD deployment (Fig 1D) did not significantly decrease compared with before (Fig 1C). These are the characteristics of an unsuccessful flow pattern.

In the posttreatment rupture case (patient 8 ), the jet flow through and into the aneurysm persisted after FD deployment; and though some stasis was observed near the top of the aneurysm 


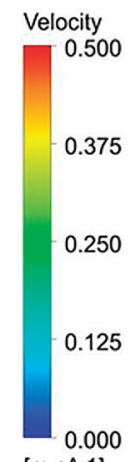

$\left[\mathrm{m} \mathrm{s} \mathrm{s}^{\wedge}-1\right]$
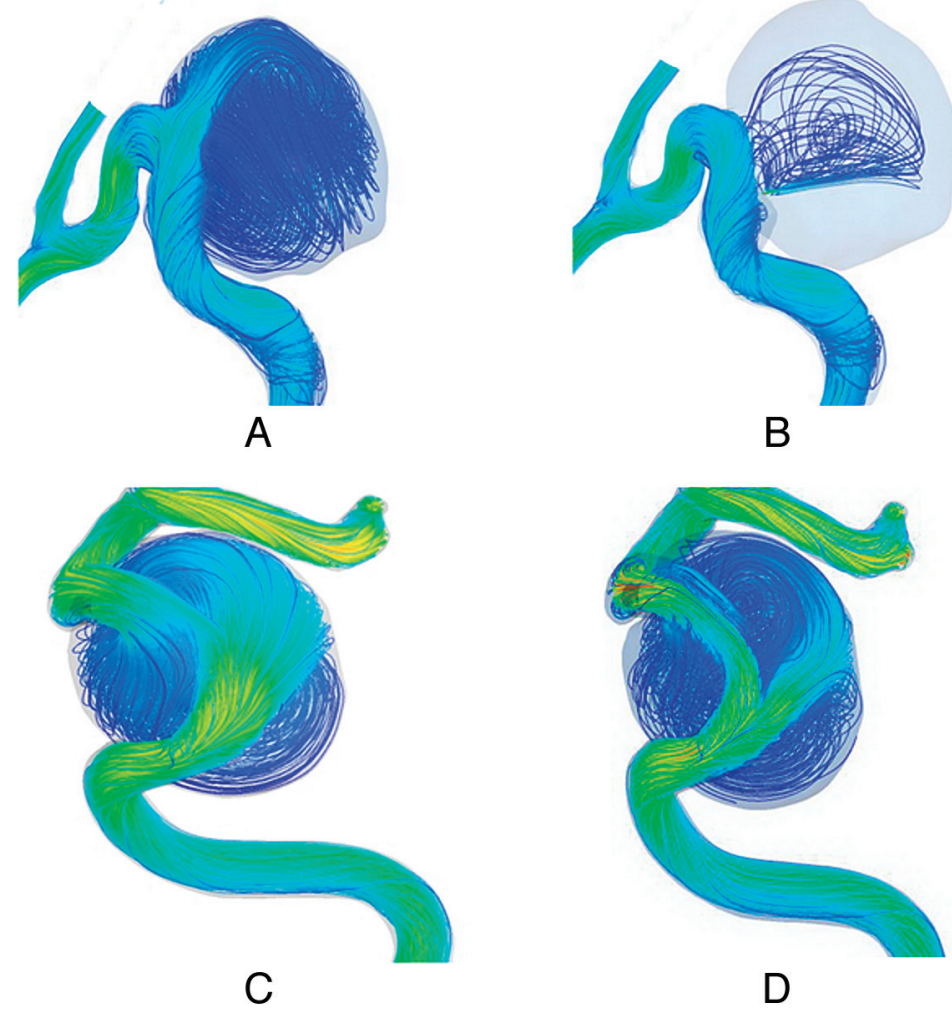

D

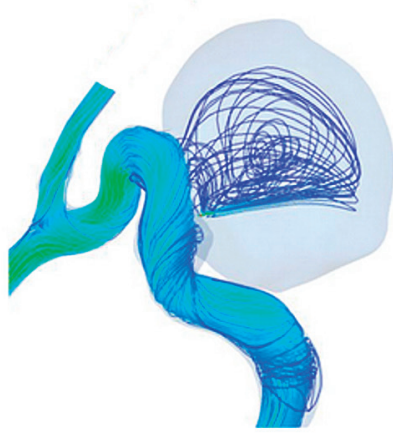

B
FIG 1. Flow pattern in patient 1 , before FD deployment $(A)$ and after FD deployment $(B)$, and in patient 6, before FD $(C)$ and after FD $(D)$ deployment.

wall, the jet continued to impact the aneurysm surface (Fig $2 B,-C$ ) in comparison with pre-FD treatment (Fig $2 A)$. This may be a contributory factor to the final rupture of the aneurysm. Following HOFD simulation, there was a marked decrease in inflow jet speed and redirection of jet flow toward the center of the FD (Fig 2D) and farther away from the peripheral aneurysm wall, a result similar to that exhibited by the successful case (Fig 1B).

For patient 6 , the jet flow continued to resemble an unsuccessful flow pattern despite application of a HOFD (Fig $3 A$ ). Even after simulation of a double HOFD (Fig $3 B$ ), the jet flow continued to resemble an unsuccessful flow pattern, though its speed was diminished (Fig 3B). The position of the largest lobule was located directly in line with a narrow jet entering the aneurysm sac, the result of a malpositioned FD (Fig 3C). Again, even after simulation of a double HOFD at the 6-month follow-up, there is still a persistent unsuccessful jet flow pattern, though diminished (Fig 3D). A 3D reconstruction of the follow-up CTA at 6 months after deployment of the first FD revealed the presence of 2 lobules (Fig 3E). A curved CTA reconstruction (Fig 3F) demonstrated that the proximal end of the malpositioned FD was partially in the aneurysm sac and only partially in the parent artery, thereby not completely covering the inflow. At 6 months, corresponding DSA of the initial FD (Fig 3G) paralleled the CFD findings, demonstrating the largest lobule; despite deployment of a second FD within the first one (Fig $3 H$ ), the lobule persisted.

For the failed case (patient 7), the jet flow persisted with the initial FD. At the 3-month follow-up, despite the aneurysm being $90 \%$ occluded, the jet flow resembled an unsuccessful pattern.
However, with the application of a double HOFD at this stage, the jet flow changed to resemble a successful pattern.

The residual aneurysm in patient 5 was treated with a covered stent (nonflow diverter) deployed within the Silk FD after 6 months. Owing to this procedure, no further correlation with clinical or imaging studies could be conducted, though simulation of a double HOFD did not reduce jet flow.

\section{Energy Loss and Volume Flow}

Figure $4 A,-B$ demonstrates the reduction of EL and VF for both successful cases and failed cases following initial FD treatment. Depending on the inflow condition, the SD values for EL were $\pm 1.2 \%$ and $\pm 10.3 \%$, while those for VF were $\pm 1.2 \%$ and $\pm 3.5 \%$, respectively, for successful and failed cases. However, the differences of average EL and VF between failed and successful cases were 16.44 times $(P<.001)$ and 5.69 times $(P<$ .001 ), respectively (Fig $4 A,-B$ ). The SD being significantly smaller than the difference in reduction between the 2 cohorts suggests that the inflow condition would have minimal influence on the EL and VF values in this study. Hence, the average values in the reduction of EL and VF of $2.6 \%$ and $8.5 \%$ for the successful cases, respectively, were considered favorable indices.

After simulation of a double HOFD (Fig $4 C,-D$ ), the average EL and VF of all failed cases were reduced to $6 \%$ (this reduces to $2.6 \%$ if patient 6 is omitted) and $10 \%$, respectively.

For patient 6 , the EL showed $100 \%$ after the initial FD, indicating that there was no reduction. The results also confirmed the flow pattern observations (Fig 3D) that double HOFD may not be sufficient to resist the jetting of blood flow into the aneurysm, thereby resulting in a higher EL (19\%). At the 6-month follow-up, patient 6 had retreatment with double FDs, but the residue remained patent 1 month afterward.

For Patient 7, EL and VF were demonstrated to be $15.3 \%$ and $51 \%$, respectively, following initial FD implantation. At 3 months, the aneurysm was reduced in size by over $90 \%$, and simulation with a double HOFD demonstrated that the EL and VF were reduced to $0.68 \%$ and $12 \%$, respectively.

For patients 6 and 8, following HOFD modifications to fully cover the aneurysm necks, EL was reduced to $49.5 \%$ and $6 \%$, respectively. VF was reduced to $37 \%$ and $28 \%$, respectively.

\section{Wall Shear Stress}

WSS was graphically mapped to the aneurysm model. The range between the highest and lowest values was very small, from 0 to 5 Pa. For successful patient treatments, the relatively high WSS regions that correlated with the jet flow before FD insertion disappeared after the deployment of FDs (patient 1, Fig 5A, - B). However, all 4 failed cases displayed high WSS before FD deployment, 


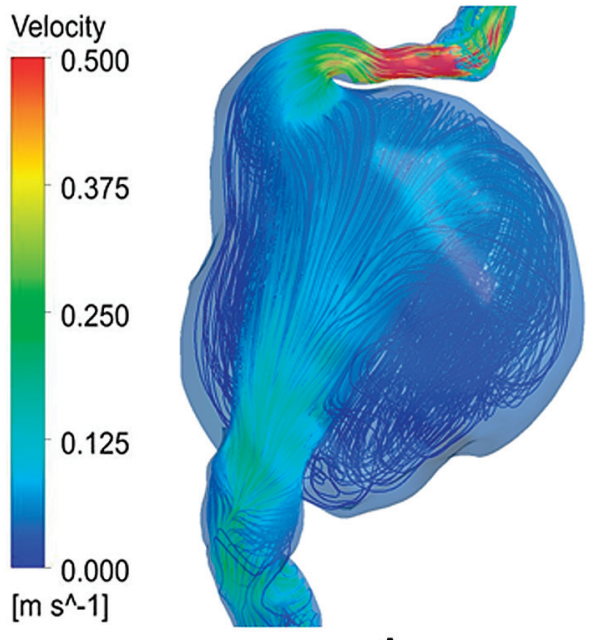

A

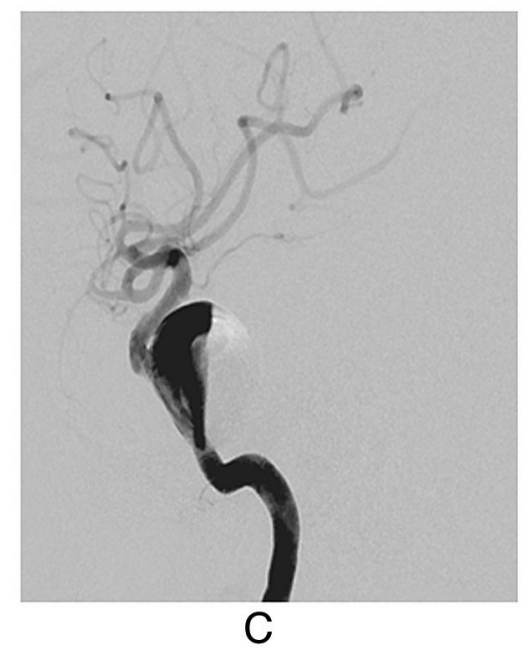

FIG 2. Flow pattern of patient 8 before $(A)$ and after FD placement $(C)$ and after placement of an HOFD (D).

which persisted after treatment with the initial FD (eg, patient 6) (Fig 5C, $-D$ ).

\section{DISCUSSION}

The porous medium simulations are based on the works of Augsburger et al, ${ }^{11}$ who concluded that this approach compared well with the real stent geometry model and allowed prediction of intra-aneurysmal flow. The effective pore size of a deployed FD would depend on the diameter and geometry of the artery, neck size of the aneurysm, and the FD size. Hence there will be variations among different aneurysms. However, in this study, we were testing the relative hemodynamic changes in the same artery/aneurysm before and after FD deployment and, in some cases, the addition of a second FD. In addition, the optimally deployed FDs should expand with a pore size to allow maximum redirection of flow from the aneurysm. Hence, we have not adjusted the porosity parameters of these FDs for the artery geometry.

Consistent with previous work in the Virtual Intracranial Stent Placement Challenge 2007, ${ }^{14}$ we showed similar therapeutic effects of the FD. Alteration of flow patterns appeared to be a remarkable differentiating feature between successful and failed treatments. The favorable flow pattern was supplemented by a marked reduction in EL, VF, and WSS within the aneurysm sac in contrast to studies showing increased aneurysm rupture risk with high WSS and EL. ${ }^{13,15}$ This result suggests that jet flow speeds have a direct proportional effect on these parameters, consistent with our previous study. ${ }^{16}$

For patient 1, a successful case, there was blood flow stasis at the near-wall region in the inferior aspect (Fig $1 B$ ) with no jet flow entering this region after the initial FD deployment, indicating the occurrence of thrombosis. This case was in contrast to all the other failed cases, in which substantial jet flow into the aneurysm sac remained after the deployment of the initial FDs, via the inflow (patients 5 and 7), through the damaged portion of the FD stent (patient 8), or from the proximal gap of the malpositioned FD (patient 6). In addition, there was a variable degree of stasis at the near-wall region. In fact, in patient 6 , we found that the location of the residual aneurysm corresponded with the direction at which the jet flow impacted (Fig 3C).

Our analysis also shed light on FD deployment strategies. For patient 8 , as the first FD was stretched and angioplastied, the pore size of the FD could have been widened and distorted, in turn reducing the ability of the FD to divert blood to the desired direction of the parent artery and allowing more flow into the aneurysm. Both our CFD results and a completion angiogram after the procedure indicated that a jet flow impinged on the anterior wall of the aneurysm at a point where later rupture occurred (Fig 2C). At the time of rupture, the angiogram showed the persisting jet flow, despite a considerable degree of thrombosis in the aneurysm. Kulcsár et $\mathrm{al}^{9}$ hypothesized that aggressive thrombosis is one factor in posttreatment rupture. Our study suggests that the interaction of unfavorable hemodynamics and thrombosis is a key part of this. The rupture was treated by vessel sacrifice. For patient 6, the first FD was malpositioned, leaving a gap between the proximal end of the FD and the aneurysm inflow. Our CFD results showed a jet flow entering the aneurysm sac through the gap, which resulted in the residual aneurysm. Even after HOFD and double HOFD, the jet flow persisted, though diminished (Fig 3); though the EL and VF were reduced, the reduction levels did not quite reach the favorable levels seen in successful treatments (Fig 4). Furthermore, the high WSS region persisted.

After the 6-month follow-up, the patient was retreated with the insertion of another FD into the first one (double FD). Despite this, the residual aneurysm lobule and jet flow persisted 1 month 


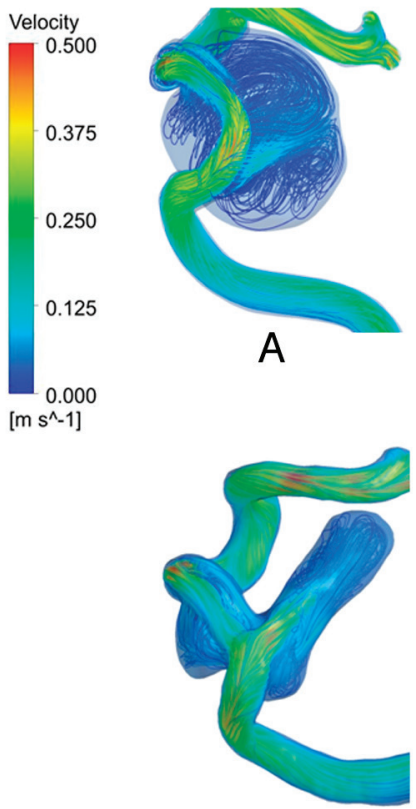

C

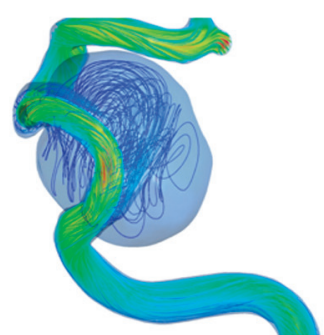

B

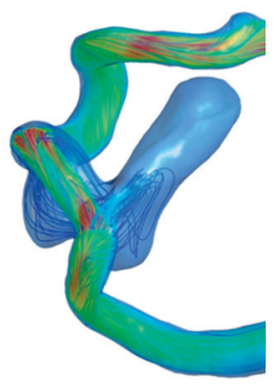

D
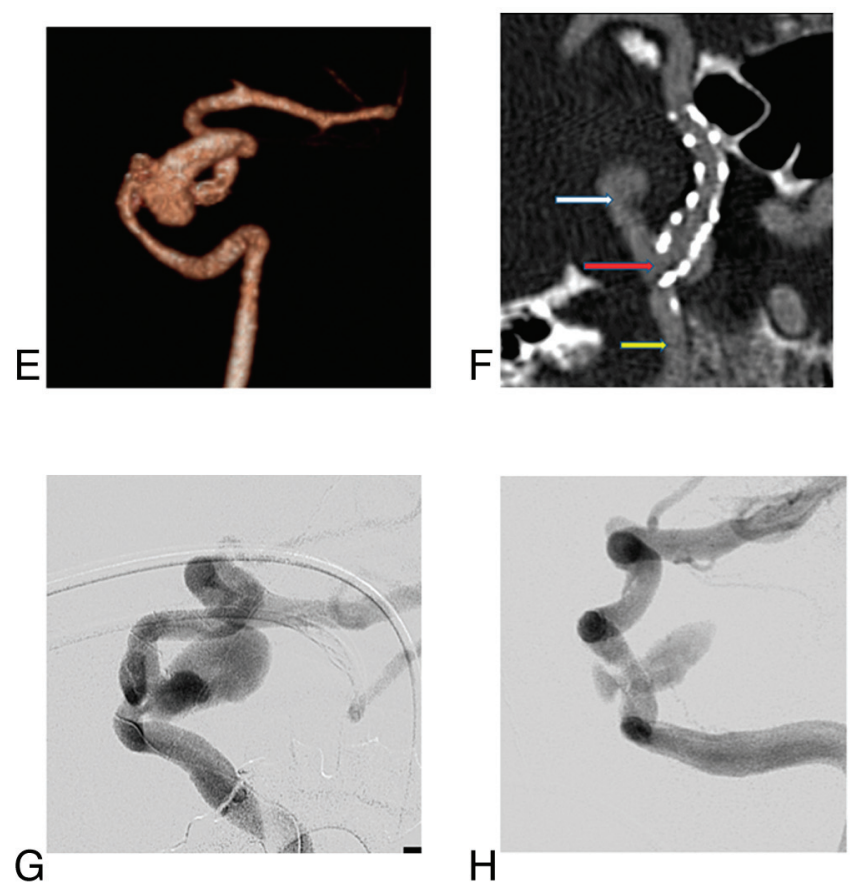

FIG 3. Flow pattern of patient 6 after placement of an HOFD $(A)$ and double HOFD (B); a residual lobule (C); and deployment of a double HOFD at follow-up (D). 3D CTA at 6 months $(E)$ shows the persisting lobules and malpositioned FD $(F)$. The white arrow indicates the persisting lobule; red arrow, the proximal end of an FD partially in the neck of the aneurysm and in the parent artery; yellow arrow, the parent artery. DSA at 6 months with the initial FD $(G)$ and after double FD deployment $(H)$.

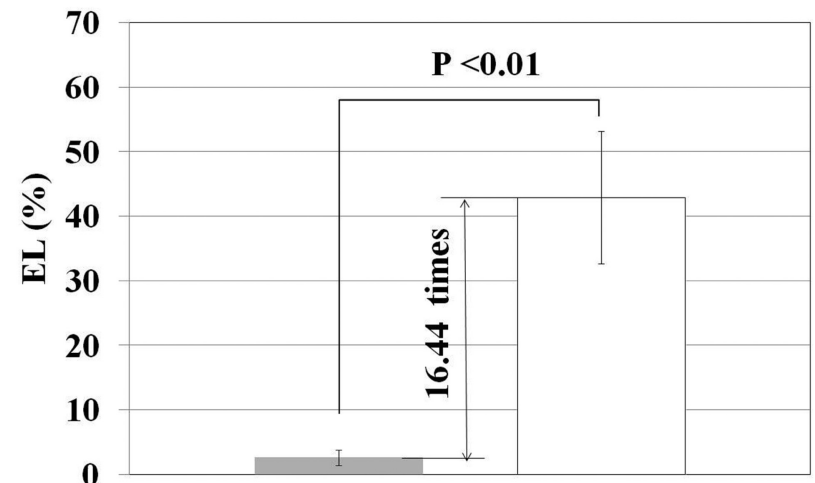

A
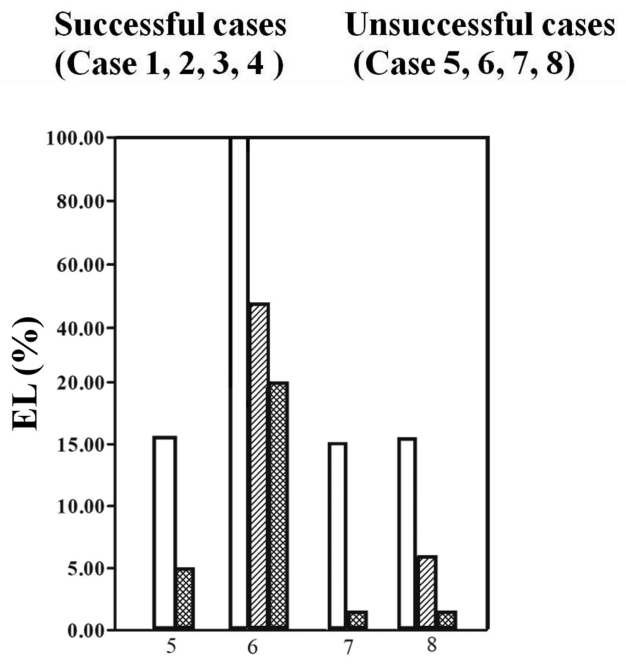

C $\square$ Initial FD HOFD Double HOFD
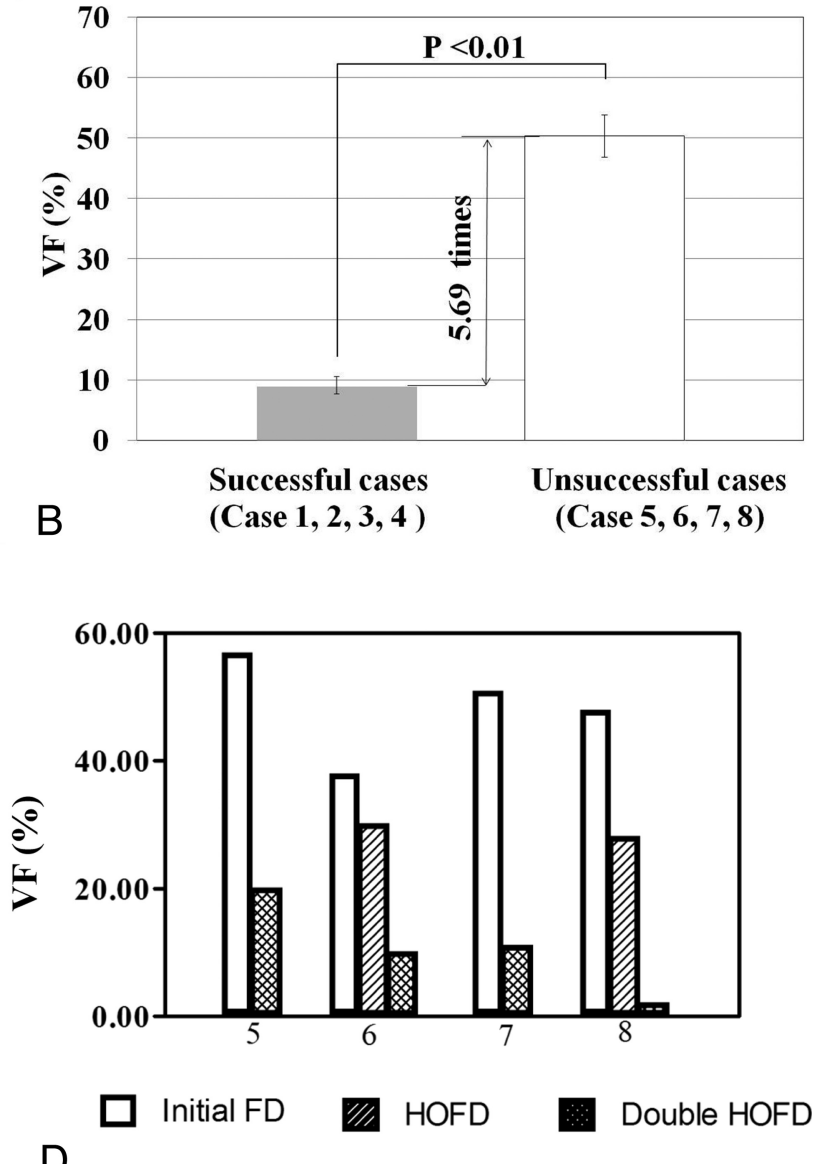

FIG 4. EL and VF results $(A$ and $B)$. HOFD and double $E L$ and $V F$ results $(C$ and $D)$. 


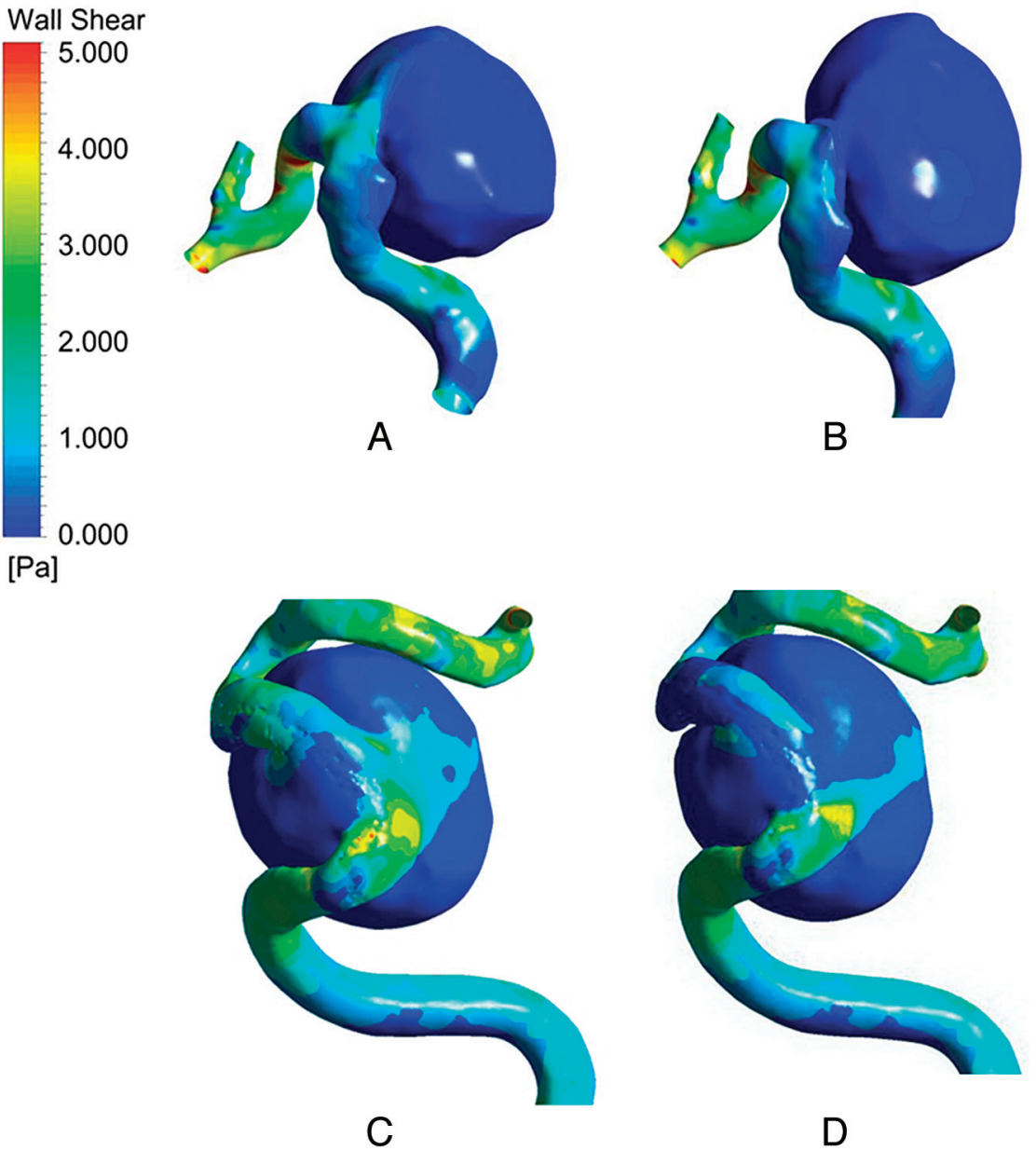

FIG 5. WSS. Patient 1 before FD deployment $(A)$ and after initial FD deployment (B). Patient 6 before $(C)$ and after $(D)$ FD deployment.

later. Just as aneurysms on straight and curved vessels may be subjected to different hemodynamics, ${ }^{12}$ the performance of an FD may vary with varying vessel curvature. ${ }^{16}$ This study suggests that the complex geometric relationship between the aneurysm neck and parent artery and the subsequent effects of this geometry on the hemodynamics after FD deployment cannot be analyzed by simple angle measurement. However, CFD can determine the resultant jet flow. It would appear in some geometry, even a double FD is not able to reverse the adverse hemodynamics (patient 6). This complex geometry may also help in understanding why the treatment was still unsuccessful in patient 5, despite the deployment of an initial intact FD in a position thought to be optimal.

For patient 7, at 3-month follow-up, areas of persistently high WSS occurred in the residual aneurysm, despite a 90\% reduction in both size and inflow into the aneurysm. Simulation with a double HOFD during this time demonstrated that the inflow jet, WSS, and EL were reduced to successful levels with a VF of $12 \%$ (just above the average successful value of $8.5 \%$ ). Patient 7 was treated with a double FD. This resulted in occlusion of the residual aneurysm, resolution of symptoms, and MR imaging changes. These findings suggested that hemodynamic factors continue to play a significant role in symptomatic aneurysms that are largely thrombosed, as asserted by Cebral et al. ${ }^{8}$

The results suggest that all parameters need to be favorable for occlusion to occur because the jet flow is a major determinant of the other parameters.

\section{Treatment Planning}

Simulation of hypothetic situations could potentially be used for treatment planning before the procedure. If the unfavorable hemodynamic results following the initial FD in patient 8 were known, we may have retreated with a double FD technique and potentially prevented rupture because double HOFD simulation resulted in favorable indices. As for case 6, unfavorable hemodynamic results, even with double FD, could indicate close imaging follow-up.

\section{Limitations}

The retrospective nature of this study and the small number of patients are limitations. We made assumptions in the initial estimations of porosities to simulate the maldeployed and damaged FDs and double HOFD. However, CFD flow patterns were very similar to the angiogram flow pattern (eg, patients 6 and 8 ), and we have validated the double HOFD porosity, ${ }^{16}$ suggesting that our assumptions are realistic. Future work, including in vitro experiments (eg, particle image velocimetry), should be performed to better simulate optimal, damaged, malpositioned and double FDs.

Other limitations include assuming that the blood vessel has a rigid arterial wall structure, models blood as a Newtonian fluid, and performs simulation under steady flow conditions. Confirming flow changes of the CFD by pre- and posttreatment angiograms may be desirable, provided the frame rate, tube angulations, and timing of injections are optimal to demonstrate the flow. If not, then it can be misleading. This work is a first approximation, and it indicates that there is good potential to better approximate the model to simulate the various parameters such as different FD geometries, sizes, and arterial geometries.

\section{CONCLUSIONS}

Patient-specific CFD, when used in conjunction with medical imaging, could help in understanding outcomes following FD deployment. Favorable hemodynamic outcomes for successful treatment of aneurysms with FDs are indicated by obliteration of the inflow jet and reduction of EL and VF and high WSS regions. Evidence collected posttreatment that points to the contrary could prompt a second intervention. We showed that a favorable treatment outcome may not have been achieved by merely placing the FD at the neck of the aneurysm sac. Instead, with FD deployment, one should consider the complex interaction among the hemodynamics, the shape of the aneurysm sac, and the geometry of the parent artery. If an optimal hemodynamic state cannot be achieved by an initial FD, a second FD within the first one (double 
FD) may be beneficial. Further evaluation by refinement of CFD modeling correlated with clinical studies and in vitro experiments, including particle image velocimetry, is suggested to confirm these results.

Disclosures: Yi Qian—RELATED: Grant: The Discovery Project is supported by the Australian Research Council.* *Money paid to the institution.

\section{REFERENCES}

1. Akpek S, Arat A, Morsi H, et al. Self-expandable stent-assisted coiling of wide-necked intracranial aneurysms: a single-center experience. AJNR Am J Neuroradiol 2005;26:1223-31

2. Molyneux AJ, Cekirge S, Saatci I, et al. Cerebral Aneurysm Multicenter European Onyx (CAMEO) trial: results of a prospective observational study in 20 European centers. AJNR Am J Neuroradiol 2004;25:39-51

3. White PM, Lewis SC, Gholkar A, et al. Hydrogel-coated coils versus bare platinum coils for the endovascular treatment of intracranial aneurysms (HELPS): a randomised controlled trial. Lancet 2011;377:1655-62

4. Wiebers DO, Whisnant JP, Huston J, et al. Unruptured intracranial aneurysms: natural history, clinical outcome, and risks of surgical and endovascular treatment. Lancet 2003;362:103-10

5. Byrne JV, Beltechi R, Yarnold JA, et al. Early experience in the treatment of intra-cranial aneurysms by endovascular flow diversion: a multicentre prospective study. PLoS One 2010;5:pii

6. Lylyk P, Miranda C, Ceratto R, et al. Curative endovascular reconstruction of cerebral aneurysms with the Pipeline embolization device: the Buenos Aires experience. Neurosurgery 2009;64:632-42, discussion 642-43, quiz N636

7. Wong GK, Kwan MC, Ng RY, et al. Flow diverters for treatment of intracranial aneurysms: current status and ongoing clinical trials. J Clin Neurosci 2011;18:737-40

8. Cebral JR, Mut F, Raschi M, et al. Aneurysm rupture following treatment with flow-diverting stents: computational hemodynamics analysis of treatment. AJNR Am J Neuroradiol 2011;32:27-33

9. Kulcsár Z, Houdart E, Bonafe A, et al. Intra-aneurysmal thrombosis as a possible cause of delayed aneurysm rupture after flow-diversion treatment. AJNR Am J Neuroradiol 2011;32:20-25

10. Appanaboyina S, Mut F, Löhner R, et al. Computational fluid dynamics of stented intracranial aneurysms using adaptive embedded unstructured grids. International Journal for Numerical Methods in Fluids 2008;57:475-93

11. Augsburger L, Reymond P, Rufenacht DA, et al. Intracranial stents being modeled as a porous medium: flow simulation in stented cerebral aneurysms. Ann Biomed Eng 2011;39:850-63

12. Meng H, Wang Z, Kim M, et al. Saccular aneurysms on straight and curved vessels are subject to different hemodynamics: implications of intravascular stenting. AJNR Am J Neuroradiol 2006;27:1861-65

13. Qian $Y$, Takao H, Fukui K, et al. Computational risk parameter analysis and geometric estimation for cerebral aneurysm growth and rupture. STROKE conference 2008, Stroke: A Journal of the American Heart Association 2008;39:527-29

14. Radaelli AG, Augsburger L, Cebral JR, et al. Reproducibility of haemodynamical simulations in a subject-specific stented aneurysm model: a report on the Virtual Intracranial Stenting Challenge 2007. J Biomech 2008;41:2069-81

15. Shojima M, Oshima M, Takagi K, et al. Magnitude and role of wall shear stress on cerebral aneurysm: computational fluid dynamic study of $\mathbf{2 0}$ middle cerebral artery aneurysms. Stroke 2004;35: 2500-05

16. Zhang Y, Chong W, Qian Y. Investigation of intracranial aneurysm hemodynamics following flow diverter stent treatment. Med Eng Phys 2013;35:608-15 